\title{
Using real-world transaction data to identify money laundering: Leveraging traditional regression and machine learning techniques
}

10.17975/sfj-2021-006

Daniel A. Harris', Kyla L. Pyndiura², Shelby L. Sturrock ${ }^{1}$, and Rebecca A.G. Christensen ${ }^{1}$

Division of Epidemiology, Dalla Lana School of Public Health, University of Toronto, Ontario, Canada

2 Faculty of Kinesiology and Physical Education, University of Toronto, Ontario, Canada

ABSTRACT: Money laundering is a pervasive legal and economic problem that hides criminal activity. Identifying money laundering is a priority for both banks and governments, thus, machine learning algorithms have emerged as a possible strategy to detect suspicious financial activity within financial institutions. We used traditional regression and supervised machine learning techniques to identify bank customers at an increased risk of committing money laundering. Specifically, we assessed whether model performance differed across varying operationalizations of the outcome (e.g., multinomial vs. binary classification) and determined whether the inclusion of investigator-derived novel features (e.g., averages across existing features) could improve model performance. We received two proprietary datasets from Scotiabank, a large bank headquartered in Canada. The datasets included customer account information $(N=4,469)$ and customers' monthly transaction histories ( $N=2,827)$ from April 15, 2019 to April 15, 2020. We implemented traditional logistic regression, logistic regression with LASSO regularization (LASSO), K-nearest neighbours (KNN), and extreme gradient boosted models (XGBoost). Results indicated that traditional logistic regression with a binary outcome, conducted with investigator-derived novel features, performed the best with an F1 score of 0.79 and accuracy of 0.72 . Models with a binary outcome had higher accuracy than the multinomial models, but the F1 scores yielded mixed results. For KNN and XGBoost, we observed little change or worsening performance after the introduction of the investigatorderived novel features. However, the investigator-derived novel features improved model performance for LASSO and traditional logistic regression. Our findings demonstrate that investigators should consider different operationalizations of the outcome, where possible, and include novel features derived from existing features to potentially improve the detection of customers at risk of committing money laundering.

KEYWORDS: money laundering, machine learning, data science, finance

\section{INTRODUCTION}

Money laundering is a sophisticated and multi-step criminal enterprise that aims to conceal the origin of funds acquired through illegal activities [1]. The United Nations estimates that $\$ 800$ billion to $\$ 2$ trillion United States (US) dollars are laundered through the global economy annually, accounting for $2-5 \%$ of global gross domestic product [1]. In the US and Canada, the Bank Secrecy Act and the Bank Act establish banks as key stakeholders in financial crime detection by holding them legally and financially culpable if their business operations fail to identify and report suspicious activity. In 2012, HSBC Bank was ordered to pay $\$ 1.9$ billion in penalties to the US government for inadequate fraud surveillance and reporting systems [2]. Therefore, effective money laundering classification and risk prediction algorithms are of great importance to both financial institutions and legal systems.

To date, only a handful of studies have leveraged machine learning techniques to identify high risk financial activity (e.g., money laundering) using real-world data [3-8]. Furthermore, across these studies, generally little attention has been paid to feature engineering and modeling approaches for varying outcome types (i.e., binary vs. multinomial classification). Chen et al. [6] provide a thorough narrative review of useful data preparation and model implementation steps when designing a risk prediction or classification algorithm for money laundering. Although the authors recommend 
creating investigator derived features such as the daily, weekly, and monthly averages of transactions [6], the impact of these features on model performance has not been widely assessed. While engineering new features from existing features may improve model performance by uncovering hidden relationships, they may also lead to overfitting and suboptimal performance. Therefore, an aim of this study is to characterize how introducing new features may affect model performance and differ across model types.

Within the current literature, we also observed that multinomial classification is relatively rare, with only two studies classifying individuals into low, medium, or highrisk categories [5, 7]. Despite banks using risk classification systems with more than two groups $[5,7]$, binary classification appears to be the default approach [3, 4, 8, 9]. Jullum et al. [7] found that multinomial classification slightly outperformed binary classification; however, the authors only reported the results from one of their models (XGBoost), citing that other models (e.g., random forest and elastic net) did not perform as well. To guide future modeling decisions for detecting suspicious financial activity, it would be valuable to characterize potential differences in multinomial vs. binary classification across multiple types of models, including parametric (e.g., regression) and non-parametric (e.g., treebased methods) approaches.

With access to individual-level data from The Bank of Nova Scotia ('Scotiabank'), a large bank in Canada, we are uniquely positioned to not only develop a classification algorithm to detect suspicious financial activity, but also address existing gaps in the literature. Our specific research objectives are to: 1) design a risk prediction algorithm to identify money laundering and 2 ) assess how different model parameterizations (e.g., type of outcome [multinomial vs. binary classification]) and inclusion of novel features impacts model performance.

\section{METHODS}

\section{Datasets and study population}

Data were obtained through a collaborative agreement between Scotiabank and the University of Toronto in December 2020. Scotiabank is among the Big 6 banks in Canada, serving over 11 million customers in Canada, over 10 million customers internationally, and ranks 3rd in market share in Canada [10]. Scotiabank provided two de-identified datasets representing a subsample of their total customer population: 1) customer account characteristics ( $N=4,469$; i.e., 'customer' dataset) and 2) customers' monthly transaction histories between April 15, 2019 to April 15, 2020 ( $N=$ 2,827; i.e., 'transaction' dataset). Customers were identified manually by an associate of Scotiabank or through the use of an internal money-laundering risk-detection algorithm; however, further details about the customer selection process were not reported by Scotiabank. The transaction dataset was aggregated to the customer level and merged one-to-one using a unique customer identification number.

\section{Outcome}

Money laundering risk status was our outcome of interest. Every customer record was reviewed manually by experts in money laundering risk alongside Scotiabank's "know your client" database. The customers were labeled as being low, medium, or at high risk of committing money laundering. However, as researchers of this study, we were not privy to the customer criteria/characteristics that constituted low, medium, or high risk. Our primary analysis considered the full multinomial outcome for three reasons: 1 ) it retained the maximum amount of information [11]; 2) dichotomizing the original three-level measure without validated cut-offs could lead to misclassification [12]; and 3) banks have previously utilized multi-group risk classification systems when flagging suspicious activity [5, 7]. To explore differences in model performance between a multinomial and binary outcome, our secondary analysis considered two binary forms of the original, three-level outcome: 1) 'low or medium risk vs. high risk' and 2) 'low risk vs. medium or high risk'.

\section{Features}

The original customer dataset contained 14 valid categorical and count features describing customer occupation, country of origin, and the number of different types of financial accounts (e.g., number of savings and chequing accounts) a customer possessed. Categorical features with $>5$ categories were collapsed to the five most common groups and the remaining values were included as "other". Customers with missing occupation and financial relationship type (e.g., co-signer, borrower, annuitant) data were retained and reassigned to a "missing" category, since missingness may be associated with suspicious financial activity. We imputed zeros for missing bank account types, as we assumed that missing 
indicated that they did not have that type of account. We also engineered novel features that contextualized potentially hidden relationships between features by calculating the relative proportions of each account type.

The transaction dataset contained 260 valid features, including monthly counts of incoming and outgoing transactions, as well as monthly sums of incoming and outgoing funds for each transaction-type (cash, debit, American Express, cheque and Visa). We imputed zeros for customers who were missing information for their transaction history under the assumption that missing represented no transactions for a given month and transaction type. Investigator-derived novel features included the monthly averages of incoming and outgoing transaction amounts by account type; yearly sums of incoming and outgoing transactions overall and by account type; and proportions of total yearly transactions (i.e., year's sum of incoming debit divided by the year's total amount of incoming funds).

Counts of the number of features included in each analysis are included in Appendix A1.

\section{Data analysis}

Descriptive statistics (chi-square for categorical data and one-way ANOVA for continuous data) and univariate plots (e.g., histograms) were used to describe the customer population and visualize the distribution of the features.

All models were conducted on four datasets: 1) the customer dataset with existing features, 2) the customer dataset with existing and investigator-derived features, 3) the merged customer and transaction datasets with existing features, and 4) the merged customer and transaction datasets with existing and investigator-derived features. Each dataset was split into training (70\%) and test (30\%) partitions, ensuring that the relative proportions of the outcome categories were approximately balanced between both. All continuous features were scaled prior to the modeling. As customers in the original datasets were expertly pre-labeled, we selected several supervised techniques to address the objectives: logistic regression with LASSO regularization (LASSO), traditional logistic regression, k-nearest neighbours (KNN), and extreme gradient boosted models (XGBoost).

\section{LASSO}

Due to the large number of features being analyzed, especially after the introduction of our novel features, we were conscious of the risk of overfitting and reduced statistical power. Thus, we opted for LASSO over ridge regression for its shrinkage and feature selection properties. We used the gImnet package in R to implement and train LASSO models (alpha =1) [13]. Cross-validation with 10-folds was conducted on the training datasets to identify the value of lambda that minimized the Brier Score.

\section{Logistic regression}

Logistic regression for binary and multinomial outcomes were specified using the g/mnet package in $\mathrm{R}$, but with the lambda parameter equal to zero (i.e., no penalty was applied to the feature weights) [13]. Traditional logistic regression was chosen as a baseline statistical method with which to compare to the other machine learning models.

\section{K-nearest neighbors (KNN)}

KNN is a non-parametric approach to classification that utilizes local patterns to determine decision boundaries between outcome groups. We used the caret package in $R$ to identify the optimal value of $k$, or number of nearestneighbors-datapoints, using 10 -fold cross-validation.

\section{Extreme gradient boosted models (XGBoost)}

We used the xgboost package to run an ensemble of decision trees [14]. Extreme gradient boosting offers a highly flexible and non-parametric approach to classification, where successive trees are trained to improve upon the performance of prior trees [15]. We used random search to test several randomly selected combinations of five unique hyperparameters $[7,16]$. One thousand sets of hyperparameters were randomly selected within their unique parameter space (learning rate, tree depth, subsample ratio of the training observations, subsample feature ratio, and leaf node splitting). 10-fold cross-validation was conducted for each unique combination of hyperparameters on the training dataset and the logistic loss function was minimized [7].

Once all models were trained, they were applied to the test dataset and compared using overall accuracy, F1 score, sensitivity, and specificity. Unlike the multinomial outcome, the default threshold for classifying the binary outcomes in the confusion matrices (i.e., predicted probability of 0.50 ) yielded poor performance given the distribution of the predicted probabilities. Therefore, we examined different 
Table 1. Baseline sociodemographic and financial account characteristics of a subsample of Scotiabank customers in April 2019/2020 overall and stratified by money laundering risk rating.

\begin{tabular}{|c|c|c|c|c|c|}
\hline & $\begin{array}{c}\text { Overall } \\
(\mathrm{N}=4469) \\
\end{array}$ & $\begin{array}{c}\text { Low risk } \\
(n=1311)\end{array}$ & $\begin{array}{c}\text { Medium risk } \\
(\mathrm{n}=1759)\end{array}$ & $\begin{array}{l}\text { High risk } \\
(\mathrm{n}=1399) \\
\end{array}$ & $p^{a, b}$ \\
\hline \multicolumn{6}{|l|}{ Country of origin, $n(\%)$} \\
\hline Canada & $3834(85.79)$ & $1241(94.66)$ & $1242(70.61)$ & 1351 (96.57) & \multirow{6}{*}{$<0.0001$} \\
\hline China & $136(3.04)$ & $7(0.53)$ & $119(6.77)$ & $10(0.72)$ & \\
\hline US & $113(2.53)$ & $37(2.82)$ & $66(3.75)$ & $10(0.72)$ & \\
\hline Mexico & $79(1.77)$ & $<5$ & $77(4.38)$ & $<5$ & \\
\hline Trinidad and Tobago & $35(0.78)$ & $<5$ & $31(1.76)$ & $<5$ & \\
\hline Other & $272(6.09)$ & $24(1.83)$ & $224(12.73)$ & $24(1.72)$ & \\
\hline \multicolumn{6}{|l|}{ Occupation code, n (\%) } \\
\hline Student & $274(6.13)$ & $64(4.88)$ & $85(4.83)$ & $125(8.93)$ & \multirow{7}{*}{$<0.0001$} \\
\hline Unemployed & $418(9.35)$ & $74(5.64)$ & $200(11.37)$ & $144(10.29)$ & \\
\hline Retired & $756(16.92)$ & $238(18.15)$ & $384(21.83)$ & $134(9.58)$ & \\
\hline Self-Employed & $616(13.78)$ & $146(11.14)$ & $268(15.24)$ & $202(14.44)$ & \\
\hline Scotiabank Other & $1054(23.58)$ & 367 (27.99) & $340(19.33)$ & $347(24.80)$ & \\
\hline Other & $1223(27.37)$ & 364 (27.77) & $457(25.98)$ & $402(28.73)$ & \\
\hline Missing & $128(2.86)$ & $58(4.42)$ & $25(1.42)$ & $45(3.22)$ & \\
\hline \multicolumn{6}{|l|}{ Relationship type, n (\%) } \\
\hline Sole & $1817(40.66)$ & $570(43.48)$ & $696(39.57)$ & 551 (39.39) & \multirow{7}{*}{$<0.0001$} \\
\hline Borrower & $350(7.83)$ & $122(9.31)$ & $116(6.59)$ & $112(8.01)$ & \\
\hline Primary Joint & $466(10.43)$ & $104(7.93)$ & $247(14.04)$ & $115(8.22)$ & \\
\hline Owner & $712(15.93)$ & $190(14.49)$ & $335(19.05)$ & $187(13.37)$ & \\
\hline Joint & $393(8.79)$ & $103(7.86)$ & $143(8.13)$ & $147(10.51)$ & \\
\hline Missing & $93(2.08)$ & $15(1.14)$ & $13(0.74)$ & $65(4.65)$ & \\
\hline Other & $638(14.28)$ & 207 (15.79) & $209(11.88)$ & $222(15.87)$ & \\
\hline \multicolumn{6}{|c|}{ Primary ownership, n (\%) } \\
\hline Yes & $3389(75.83)$ & 1005 (76.66) & $1391(79.08)$ & $993(70.98)$ & \multirow{3}{*}{$<0.0001$} \\
\hline No & 987 (22.09) & $291(22.20)$ & $355(20.18)$ & $341(24.37)$ & \\
\hline Missing & $93(2.08)$ & $15(1.14)$ & $13(0.74)$ & $65(4.65)$ & \\
\hline \multicolumn{6}{|l|}{ Accounts, mean (SD) ${ }^{b}$} \\
\hline Chequing & $0.45(0.74)$ & $0.33(0.65)$ & $0.60(0.87)$ & $0.39(0.59)$ & 0.0715 \\
\hline Credit cards & $0.49(0.90)$ & $0.57(0.92)$ & $0.43(0.90)$ & $0.48(0.88)$ & 0.0100 \\
\hline Loans & $0.23(0.54)$ & $0.28(0.58)$ & $0.19(0.52)$ & $0.24(0.52)$ & 0.0381 \\
\hline Mortgages & $0.09(0.38)$ & $0.09(0.42)$ & $0.09(0.38)$ & $0.10(0.36)$ & 0.8420 \\
\hline Savings & $0.35(0.87)$ & $0.31(0.79)$ & $0.41(1.01)$ & $0.31(0.75)$ & 0.9080 \\
\hline Safety deposit boxes & $0.03(0.17)$ & $0.03(0.17)$ & $0.03(0.18)$ & $0.03(0.17)$ & 0.9680 \\
\hline Term deposits & $0.25(0.61)$ & $0.26(0.60)$ & $0.30(0.69)$ & $0.19(0.51)$ & 0.0017 \\
\hline
\end{tabular}

$\mathrm{N}=$ sample size; $\%$ = percent; $\mathrm{SD}=$ standard deviation; cells with fewer than 5 individuals have been suppressed.

a Chi-square approximation was used when cell sizes were less than 5 .

${ }^{\mathrm{b}}$ ANOVA were used as a global test of mean differences across money laundering risk status. 
thresholds ranging from 0.05 to 0.75 , increasing by 0.05 increments. Thresholds of 0.30 for 'low or medium risk vs. high risk' and 0.70 for 'low risk vs. medium or high risk' maximized sensitivity and specificity for both outcomes and was used throughout. All analyses were conducted in R Version 4.0.4 (Boston, United States) [17].

\section{RESULTS}

Table 1 includes a description of customers sociodemographic and account information overall and stratified by money laundering risk status. We observed significant differences $(p<0.05)$ in occupation, country of origin, and number of different account types across money laundering risk groups. For example, there was a greater proportion of students and unemployed customers in the medium- and high-risk categories compared to customers in the low-risk category.

\section{Best performing models}

Figure 1 depicts the F1 score and Figure 2 depicts the accuracy of each model by dataset and type of outcome. Logistic regression conducted on the merged customer and transaction datasets with investigator-derived features, and with the binary outcome 'low risk vs. medium or high risk', preformed the best with an F1 score $=0.79$, accuracy $=0.72$, sensitivity $=0.71$, and specificity $=0.74$ (Table 2 ). Multinomial logistic regression conducted on the same dataset performed second best with an F1 score $=0.79$, accuracy $=0.65$, sensitivity: low risk $=0.56$; medium risk $=0.72$; high risk $=0.63$, and specificity: low risk $=0.85$; medium risk $=0.79$; high risk $=$ 0.82 (Table 3 ). LASSO conducted on the merged customer and transaction datasets with investigator-derived features, and with the binary outcome 'low risk vs. medium or high risk', was the third best model with an F1 score $=0.69$, accuracy $=0.62$, sensitivity $=0.58$, and specificity $=0.72$ (Table 2). Appendix A2 reports the model diagnostics for the binary outcome 'low or medium risk vs. high risk', which generally performed the same or worse than the other binary outcome. KNN and XGBoost performed moderately well, but poorer than LASSO and logistic regression, with F1 scores not exceeding 0.70 (Appendix A3-A5).

\section{Multinomial vs. binary outcomes}

The binary models performed better, on average, than the multinomial models in terms of overall accuracy, regardless of how the outcome was categorized (i.e., 'low risk vs. medium or high risk' or 'low or medium risk vs. high risk') (Figure 2). For example, the binary logistic regression models conducted on the merged customer and transaction dataset with investigator-derived features had the highest accuracy of $\mathbf{0 . 7 2}$. This represents an absolute increase of $7 \%$ in accuracy compared to the multinomial logistic model using the same dataset. A different pattern was observed for the F1 score (Figure 1), which depended on the type of model and outcome examined. The multinomial LASSO and logistic regression models outperformed the binary models when using the customer only data and the customer data including the investigator-derived features. The models performed similarly when using the transaction dataset.

\section{Impact of novel features}

For the KNN models, there was little change in the overall accuracy and F1 score with the addition of the investigatorderived features. For XGBoost, a similar pattern was observed. Introducing investigator-derived features had minimal impact on the overall accuracy and F1 scores; however, when they

Table 2. Accuracy, F1 Score, Sensitivity and Specificity for LASSO and logistic regression with binary outcome 'low risk vs. medium or high risk.'

\begin{tabular}{lcccccccc}
\hline \multirow{2}{*}{ Dataset } & \multicolumn{2}{c}{ Accuracy } & \multicolumn{2}{c}{ F1 } & \multicolumn{2}{c}{ Sensitivity } & \multicolumn{2}{c}{ Specificity } \\
\cline { 2 - 9 } & LASSO & Logistic & LASSO & Logistic & LASSO & Logistic & LASSO & Logistic \\
\hline Customer & 0.54 & 0.56 & 0.59 & 0.61 & 0.48 & 0.49 & 0.71 & 0.72 \\
$\begin{array}{l}\text { Customer + novel } \\
\text { features }\end{array}$ & 0.56 & 0.56 & 0.61 & 0.61 & 0.49 & 0.49 & 0.73 & 0.72 \\
$\begin{array}{l}\text { Customer + } \\
\text { transaction }\end{array}$ & 0.58 & 0.67 & 0.64 & 0.74 & 0.52 & 0.65 & 0.72 & 0.72 \\
$\begin{array}{l}\text { Customer + } \\
\text { transaction + novel } \\
\text { features }\end{array}$ & 0.62 & 0.72 & 0.69 & 0.79 & 0.58 & 0.71 & 0.72 & 0.74 \\
\hline
\end{tabular}



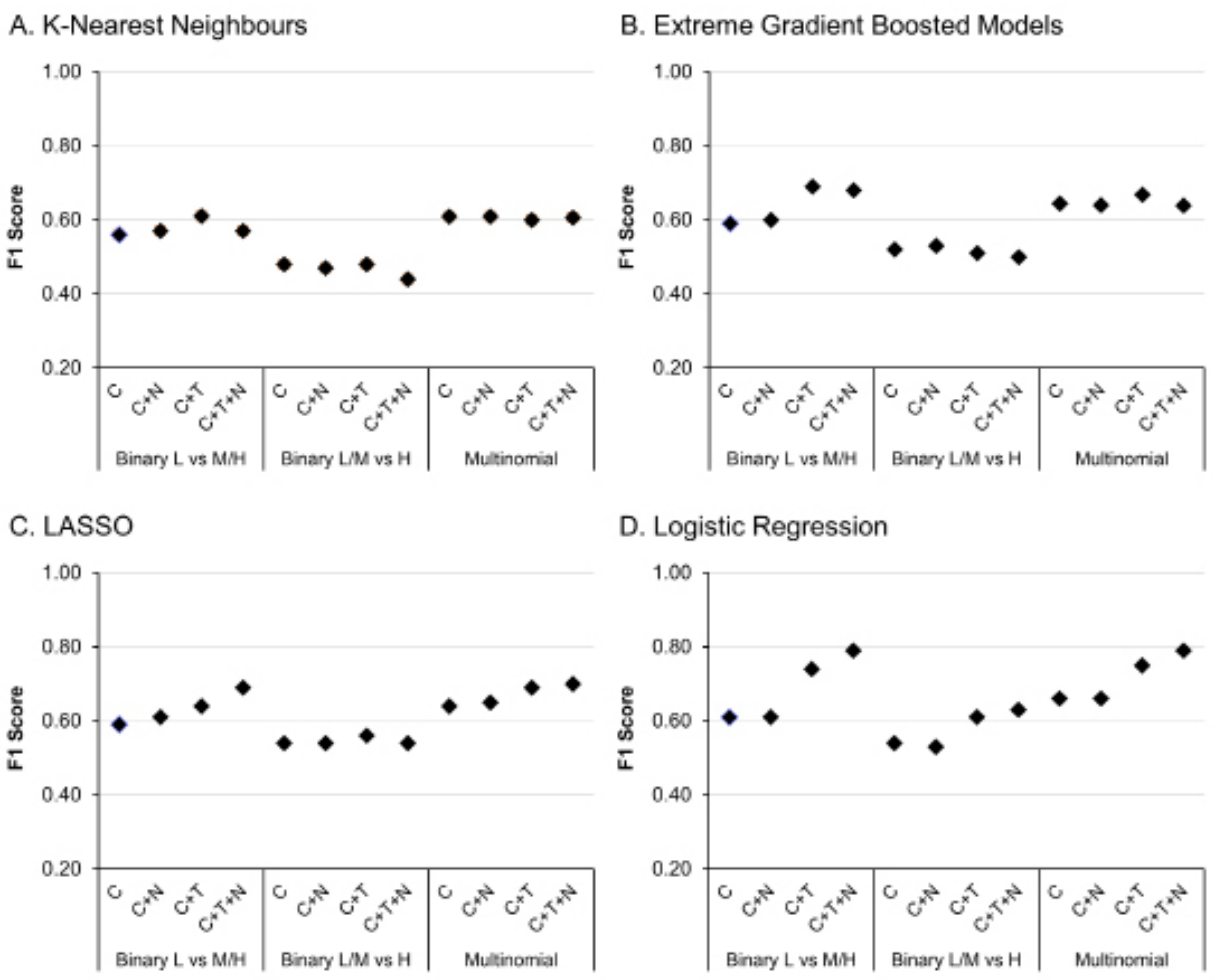

Figure 1. Model performance (F1 score) for classification algorithms identifying money laundering risk. The $y$-axis represents $\mathrm{F} 1$ score. The $\mathrm{x}$-axis represents the dataset $(\mathrm{C}=$ customer dataset with existing features; $\mathrm{C}+\mathrm{N}=$ customer dataset with existing and novel features; $\mathrm{C}+\mathrm{T}=$ merged customer and transaction datasets with existing features; $\mathrm{C}+\mathrm{T}+\mathrm{N}=$ merged customer and transaction datasets with existing and novel features) and outcome type (Binary $\mathrm{L}$ vs $\mathrm{M} / \mathrm{H}=$ binary categorization with low risk vs. medium or high risk; Binary L/M vs. $\mathrm{H}=$ binary categorization with low or medium risk vs. high risk; Multinomial = low, medium and high risk modeled as separate categories).

were added to the merged customer and transaction dataset for the multinomial models, model performance worsened by $3 \%$ for accuracy and 0.03 for F1 score. Across the datasets, there is a clear gradient of increasing performance with the LASSO and logistic regression models, such that introducing additional (i.e., transaction) and investigatorderived features improved the F1 score. For example, the F1 score for binary logistic regression ('low risk vs. medium or high risk') had an absolute increase of 0.05 , or a relative increase of $7 \%$ (i.e., $0.79 / 0.74$ ) after the introduction of investigator-derived features alone (Table 2).

\section{DISCUSSION}

The primary aim of our research was to design an algorithm to identify individuals at an increased risk of committing money laundering. Logistic regression with a binary outcome ('low risk vs. medium or high risk'), conducted on the merged customer and transaction datasets with additional investigator-derived features, was the best performing model with an F1 score of 0.79 and overall accuracy of 0.72 . The secondary aims of this research were to compare whether outcome type (binary vs. multinomial) and the addition of investigator-derived features (e.g., relative and other summary measures) improved performance. Overall, the results indicated that while the binary outcomes modestly outperformed the multinomial outcomes, the final choice of outcome depends on what categorizations are used (i.e., 'low or medium risk vs. high risk'; 'low risk vs. medium or high risk') and the goals of the classification task. Lastly, we observed that the addition of investigator-derived features led to gains in model performance, but these results were mixed across model types.

Due to lack of research and inconsistent use of performance metrics, it is challenging to determine how our models compare to other studies. Our best performing model had a sensitivity of $71 \%$ and specificity of $74 \%$, indicating relatively 
A. K-Nearest Neighbours

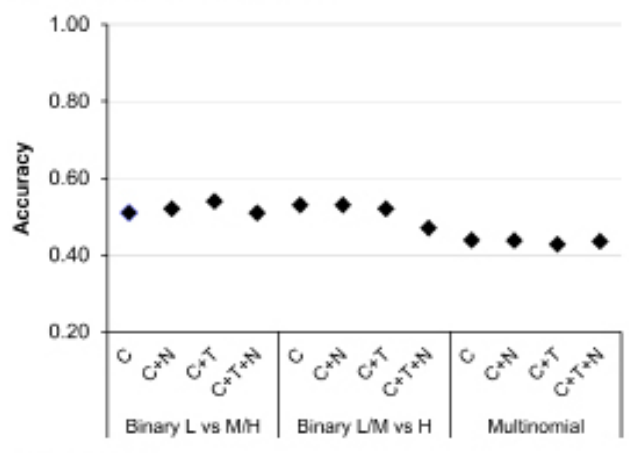

C. LASSO

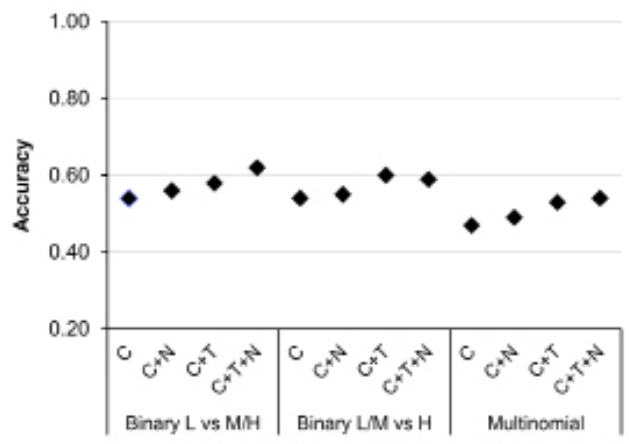

B. Extreme Gradient Boosted Models

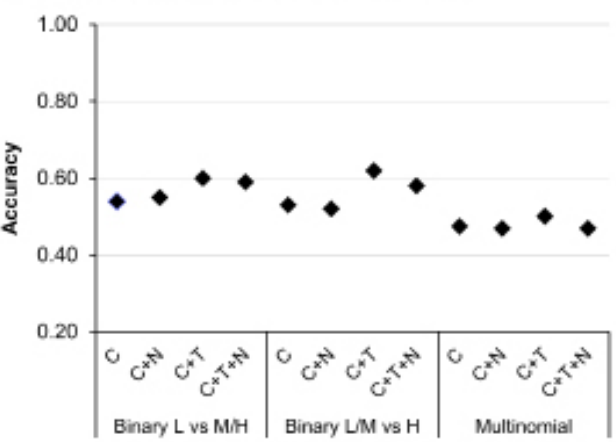

D. Logistic Regression

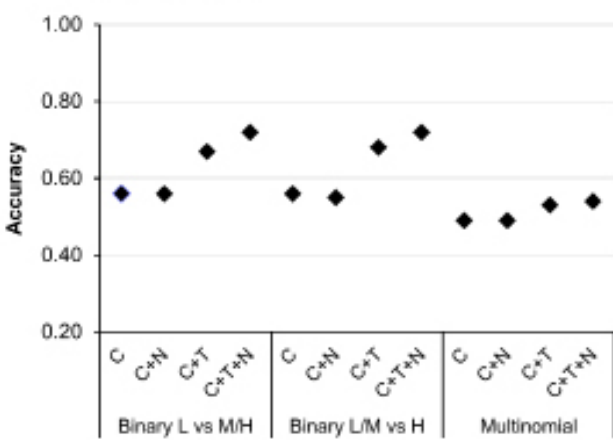

Figure 2. Model performance (accuracy) for classification algorithms identifying money laundering risk. The $y$-axis represents accuracy. The $\mathrm{x}$-axis represents the dataset $(\mathrm{C}=$ customer dataset with existing features; $\mathrm{C}+\mathrm{N}=\mathrm{customer}$ dataset with existing and novel features; $\mathrm{C}+\mathrm{T}=$ merged customer and transaction datasets with existing features; $\mathrm{C}+\mathrm{T}+\mathrm{N}=$ merged customer and transaction datasets with existing and novel features) and outcome type (Binary $\mathrm{L}$ vs. $\mathrm{M} / \mathrm{H}=$ binary categorization with low risk vs. medium or high risk; Binary L/M vs. $\mathrm{H}=$ binary categorization with low or medium risk vs. high risk; Multinomial = low, medium and high risk modeled as separate categories).

Table 3. Accuracy, F1 Score, Sensitivity and Specificity for LASSO and logistic regression with multinomial outcome.

\begin{tabular}{|c|c|c|c|c|c|c|c|c|}
\hline \multirow{2}{*}{ Dataset } & \multicolumn{2}{|c|}{ Accuracy } & \multicolumn{2}{|c|}{ F1 } & \multicolumn{2}{|c|}{ Sensitivity } & \multicolumn{2}{|c|}{ Specificity } \\
\hline & LASSO & Logistic & LASSO & Logistic & LASSO & Logistic & LASSO & Logistic \\
\hline \multirow[t]{3}{*}{ Customer } & & & & & L: 0.32 & L: 0.35 & L: 0.78 & L: 0.78 \\
\hline & 0.47 & 0.49 & 0.64 & 0.66 & M: 0.60 & M: 0.59 & M: 0.68 & M: 0.70 \\
\hline & & & & & $\mathrm{H}: 0.46$ & H: 0.49 & H: 0.75 & H: 0.75 \\
\hline Customer + & & & & & L: 0.36 & L: 0.38 & L: 0.76 & L: 0.74 \\
\hline \multirow[t]{2}{*}{ novel features } & 0.49 & 0.49 & 0.65 & 0.66 & M: 0.60 & M: 0.58 & M: 0.68 & M: 0.71 \\
\hline & & & & & $\mathrm{H}: 0.46$ & $\mathrm{H}: 0.48$ & $\mathrm{H}: 0.78$ & H: 0.78 \\
\hline Customer + & & & & & L: 0.30 & L: 0.52 & L: 0.87 & L: 0.83 \\
\hline \multirow[t]{2}{*}{ transaction } & 0.53 & 0.60 & 0.69 & 0.75 & M: 0.71 & M: 0.64 & M: 0.66 & M: 0.78 \\
\hline & & & & & $\mathrm{H}: 0.51$ & $\mathrm{H}: 0.63$ & H: 0.75 & H: 0.79 \\
\hline Customer + & & & & & L: 0.25 & L: 0.56 & L: 0.90 & L: 0.85 \\
\hline transaction + & 0.54 & 0.65 & 0.70 & 0.79 & M: 0.74 & M: 0.72 & M: 0.65 & M: 0.79 \\
\hline novel features & & & & & $\mathrm{H}: 0.54$ & H: 0.63 & $\mathrm{H}: 0.74$ & H: 0.82 \\
\hline
\end{tabular}

$\mathrm{L}=$ low risk; $\mathrm{M}=$ =medium risk; $\mathrm{H}=$ high risk of money laundering. 
good identification of individuals with medium or high risk of committing money laundering compared to the expertly defined gold standard. There is generally little guidance on what constitutes low, moderate, or high sensitivity and specificity, because this ultimately reflects a value judgement by the end user. In medicine and clinical epidemiology, diagnostic tests with a sensitivity and specificity $\geq 90 \%$ are typically considered highly accurate [18], but this too varies according to the outcome, available technology, and overall usefulness of the tool to clinicians and public health practitioners. Our final model with $71 \%$ sensitivity may improve existing classification systems, especially when used in concert with other tools; however, data regarding the model's impact on real-world decisions would be required to determine its usefulness.

We found that the type of outcome mattered substantially across all model diagnostics. We compared a multinomial outcome to two binary outcomes and determined whether different cut-offs for the binary outcomes led to changes in model performance. In general, the multinomial performed similarly to the binary outcome with a 'low risk vs. medium or high risk' of money laundering categorization, which produced the model with the highest performance (i.e., traditional logistic regression using 'low risk vs. medium or high risk' as the outcome conducted on the merged customer and transaction dataset with investigator-derived features had the highest performance [F1=0.79]). Importantly, both models performed substantially better than the binary models with a 'low or medium risk vs. high risk' of money laundering categorization. We hypothesize that this discrepancy may be due to the fact that customers expertly classified as medium or high risk share common characteristics or behaviours, and thus collapsing low and medium risk together may have made it challenging to discriminate binary classes in this form. Furthermore, although the binary logistic regression model with the 'low risk vs. medium or high risk' categorization was the best performing model, we note that there are important implications to its real-world use. Mainly, there are millions of transactions that pass through a bank each day, therefore, minimizing false positives is important [19]. Using a binary outcome with this categorization may result in a greater number of customers being flagged as having suspicious activity (i.e., both the medium and high-risk records are flagged with this model). Consequently, if the ultimate goal is to identify customers with the greatest risk of committing money laundering, then a multinomial outcome should be utilized when available.

We also found that introducing investigator-derived features can substantially improve model performance for some types of models, but not all. We conceptualized identifying money laundering as a missing data problem, and thus engineered relationships between features as a strategy to characterize hidden relationships. We built upon Zhang et al. [3] by providing additional empirical support for introducing novel features to augment existing data. However, we note that the impact of additional features varies based on the modeling approach. Perhaps unsurprisingly, we found that KNN and XGBoost did not improve or performed slightly worse after introducing our novel features. Non-parametric, and decision trees in particular, are known to have overfitting issues [3]. However, LASSO and logistic regression observed notable boosts in model performance after introducing investigator-derived features.

To our knowledge, this is the first study to use real-world data to empirically examine whether investigator-derived features improve model performance over existing features alone when classifying money laundering risk. These features are relatively simple to engineer and may boost (parametric) model performance. Also, having access to a three-level, expert rating for money laundering risk is also a notable strength of current work. However, there are some limitations. First, the XGBoost models were computationally intensive, with one model requiring several days to complete. Given the required computing time, we were unable to explore a greater number of unique hyperparameter sets (e.g., 10,000 vs. 1,000). We also included several hundred features in our traditional regression models despite having a relatively small sample size. As such, a reduction in statistical power is likely in this context. Last, we acknowledge that the datasets and money laundering risk status used in this study were created and identified using internal processes and algorithms within Scotiabank. As authors of this study, we are not privy to Scotiabank's internal methods, and thus cannot provide a comprehensive analysis on how their customer selection and labeling procedures could impact our findings. However, as the customer sample used in the current study was selected based on an internal, money-laundering risk-detection algorithm and expert review, we suspect that this may lend to improved model performance compared to a customer 
sample without such selection. In other words, we anticipate that the customers analyzed here do not reflect the broader Scotiabank customer population, but a refined sample with a more easily delineated risk of money laundering.

\section{CONCLUSION}

With access to individual-level, real-world banking data, we tested several algorithms to classify money laundering risk and examined how various model parametrizations impacted model performance. This current work is unique and important especially when acknowledging the fact that such data sources are rarely available for research and publication. We found that traditional logistic regression largely outperformed supervised machine learning models in identifying suspicious financial activity. Future studies and analysts should explore different outcome categorizations and assess how this may impact model performance. Lastly, we observed that engineering features from existing features can boost model performance, but that it may not benefit all model types. Future work should explore how these model specifications can improve the detection of suspicious final activity in real-world contexts.

\section{ACKNOWLEDGEMENTS}

We would like to acknowledge the 2021 IMI Big Data and Artificial Intelligence Case Competition for giving us the opportunity to prepare this work. We specifically acknowledge Professor Kevin Yousie, Dr. Mark Engel, Professor Soo Min Toh, Dr. Simon Liu, Dr. Duncan Smith-Halverson, and Dr. Sacha Noukhovitch for their roles in facilitating the competition. Last, we acknowledge all the competing teams, applaud their successes, and express our gratitude for being able to participate in the competition alongside them.

\section{REFERENCES}

1. United Nations Office on Drugs and Crime. Money Laundering [Internet]. [cited: 2021 Mar 13]. Available from: https://www.unodc.org/unodc/en/moneylaundering/overview.html.

2. HSBC's \$1.9B money laundering settlement approved by judge [Internet]. Toronto, ON; 2013 [cited $2021 \mathrm{Mar}$ 13]. Available from: https://www.cbc.ca/news/business/ hsbc-s-1-9b-money-laundering-settlement-approved-byjudge-1.1377272.

3. Zhang Y, Trubey P. Machine Learning and Sampling Scheme: An Empirical Study of Money Laundering Detection. Computational Economics. 2019;54(3):1043-

\section{3. https://doi.org/10.1007/s10614-018-9864-z.}

4. Savage D, Wang Q, Chou P, Zhang X, Yu X. Detection of money laundering groups using supervised learning in networks. arXiv preprint arXiv:160800708. 2016.

5. Wang S-N, Yang J-G, editors. A money laundering risk evaluation method based on decision tree. 2007 International Conference on Machine Learning and Cybernetics. 2007; 283-286. doi: 10.1109/ ICMLC.2007.4370155.

6. Chen Z, Van Khoa LD, Teoh EN, Nazir A, Karuppiah EK, Lam KS. Machine learning techniques for anti-money laundering (AML) solutions in suspicious transaction detection: a review. Knowledge and Information Systems. 2018;57(2):245-85. https://doi.org/10.1007/ s10115-017-1144-z.

7. Jullum M, Løland A, Huseby RB, Ånonsen G, Lorentzen J. Detecting money laundering transactions with machine learning. Journal of Money Laundering Control. 2020; 23(1): 173-186. https://doi.org/10.1108/JMLC-07-20190055 .

8. Tang J, Yin J. Developing an intelligent data discriminating system of anti-money laundering based on SVM. 2005 International conference on machine learning and cybernetics. 2005; (6): 3453-3457. doi: 10.1109/ICMLC.2005.1527539.

9. Nerurkar P, Bhirud S, Patel D, Ludinard R, Busnel Y, Kumari S. Supervised learning model for identifying illegal activities in Bitcoin. Applied Intelligence. 2021; (51): 3824-3843. https://doi.org/10.1007/s10489-02002048-w.

10. Scotiabank. 2020 Annual Repor [Internet]. 2020. [cited 2021 September 20]. Available from: https://www. scotiabank.com/content/dam/scotiabank/corporate/ quarterly-reports/2020/q4/BNS_Annual_Report_2020.pdf.

11. Zhao LP, Kolonel LN. Efficiency Loss from Categorizing Quantitative Exposures into Qualitative Exposures in Case-Control Studies. American Journal of Epidemiology. 1992;136(4):464-74. https://doi.org/10.1093/ oxfordjournals.aje.a116520.

12. Rothman KJ, Greenland S, Lash TL. Modern epidemiology: Lippincott Williams \& Wilkins; 2008.

13. Friedman J, Hastie T, Tibshirani R. Regularization Paths for Generalized Linear Models via Coordinate Descent. J Stat Softw. 2010;33(1):1-22.

14. Chen T, Guestrin C. Xgboost: A scalable tree boosting system. Proceedings of the $22 \mathrm{nd} \mathrm{acm}$ sigkdd international conference on knowledge discovery and data mining. 2016; 785-794. doi: 10.1145/2939672.2939785.

15. Natekin A, Knoll A. Gradient boosting machines, a tutorial. Frontiers in neurorobotics. 2013;7:21. https:// doi.org/10.3389/fnbot.2013.00021.

16. Bergstra J, Bardenet R, Bengio Y, Kégl B. Algorithms for hyper-parameter optimization. 25th annual conference on neural information processing systems (NIPS 2011). 
Neural Information Processing Systems Foundation. 2011; 2546-2554.

17. R Core Team. R: A Language and Environment for Statistical Computing. Vienna, Austria: R Foundation for Statistical Computing 2020 [cited: 2021 Mar 13]. Available from: https://www.R-project.org/.

18. Stephan BC, Kurth T, Matthews FE, Brayne C, Dufouil C. Dementia risk prediction in the population: are screening models accurate? Nat Rev Neurol. 2010;6(6):318-26. doi: 10.1038/nrneurol.2010.54.

19. Grint R, O'Driscoll C, Patton S. New technologies and anti-money laundering compliance report. 2017. Available from: https://www.fca.org.uk/publications/ research/new-technologies-and-anti-money-launderingcompliance-report. 


\section{APPENDIX A}

Table A1. Sample size, unique features, and total features for all datasets.

\begin{tabular}{|c|c|c|c|}
\hline Dataset & Sample size & Unique features $^{a}$ & Total columns $^{b}$ \\
\hline Customer & 4,469 & 14 & 31 \\
\hline $\begin{array}{l}\text { Customer + investigator } \\
\text { derived features }\end{array}$ & 4,469 & 22 & 39 \\
\hline Customer + transaction & 2,827 & 274 & 289 \\
\hline $\begin{array}{l}\text { Customer }+ \text { transaction }+ \\
\text { investigator derived features }\end{array}$ & 2,827 & 456 & 471 \\
\hline
\end{tabular}

a Unique features represent a single feature (i.e., variable or column) within the datasets that contain a defined characteristic (e.g., occupation or number of savings accounts).

${ }^{\mathrm{b}}$ Total columns reflects the model matrix after the categorical variables have been converted into dummy variables. These are the number of features that were entered into each model to classify money laundering risk.

Table A2. Accuracy, F1 score, sensitivity and specificity for LASSO and logistic regression with binary outcome 'low or medium risk vs. high risk' of money laundering.

\begin{tabular}{lcccccccc}
\hline \multirow{2}{*}{ Dataset } & \multicolumn{2}{c}{ Accuracy } & \multicolumn{2}{c}{ F1 } & \multicolumn{2}{c}{ Sensitivity } & \multicolumn{2}{c}{ Specificity } \\
\cline { 2 - 9 } & LASSO & Logistic & LASSO & Logistic & LASSO & Logistic & LASSO & Logistic \\
\hline Customer & 0.54 & 0.56 & 0.54 & 0.54 & 0.85 & 0.83 & 0.41 & 0.43 \\
Customer + novel features & 0.55 & 0.55 & 0.54 & 0.53 & 0.84 & 0.81 & 0.42 & 0.43 \\
Customer + transaction & 0.60 & 0.68 & 0.56 & 0.61 & 0.81 & 0.77 & 0.50 & 0.64 \\
$\begin{array}{l}\text { Customer + transaction + } \\
\text { novel features }\end{array}$ & 0.59 & 0.72 & 0.54 & 0.63 & 0.77 & 0.78 & 0.51 & 0.69 \\
\hline
\end{tabular}

Table A3. Accuracy, F1 score, sensitivity and specificity for k-nearest neighbours and extreme gradient boosted models with the binary outcome 'low or medium risk vs. high risk.'

\begin{tabular}{lcccccccc}
\hline \multirow{2}{*}{ Dataset } & \multicolumn{2}{c}{ Accuracy } & \multicolumn{2}{c}{ F1 } & \multicolumn{2}{c}{ Sensitivity } & \multicolumn{2}{c}{ Specificity } \\
\cline { 2 - 9 } & KNN & XGBoost & KNN & XGBoost & KNN & XGBoost & KNN & XGBoost \\
\hline $\begin{array}{l}\text { Customer } \\
\begin{array}{l}\text { Customer + novel } \\
\text { features }\end{array}\end{array}$ & 0.53 & 0.53 & 0.48 & 0.52 & 0.70 & 0.81 & 0.45 & 0.41 \\
$\begin{array}{l}\text { Customer + } \\
\text { transaction }\end{array}$ & 0.53 & 0.52 & 0.47 & 0.53 & 0.67 & 0.85 & 0.46 & 0.38 \\
$\begin{array}{l}\text { Customer + } \\
\text { transaction + novel } \\
\text { features }\end{array}$ & 0.47 & 0.58 & 0.44 & 0.50 & 0.65 & 0.65 & 0.45 & 0.55 \\
\hline
\end{tabular}

KNN=K-nearest neighbours; XGBoost=Extreme Gradient Boosted Models. 
Table A4. Accuracy, F1 score, sensitivity and specificity for k-nearest neighbours and extreme gradient boosted models with the binary outcome 'low risk vs. medium or high risk.'

\begin{tabular}{lcccccccc}
\hline \multirow{2}{*}{ Dataset } & \multicolumn{2}{c}{ Accuracy } & \multicolumn{2}{c}{ F1 } & \multicolumn{2}{c}{ Sensitivity } & \multicolumn{2}{c}{ Specificity } \\
\cline { 2 - 9 } & KNN & XGBoost & KNN & XGBoost & KNN & XGBoost & KNN & XGBoost \\
\hline $\begin{array}{l}\text { Customer } \\
\begin{array}{l}\text { Customer + novel } \\
\text { features }\end{array}\end{array}$ & 0.51 & 0.54 & 0.56 & 0.59 & 0.44 & 0.47 & 0.69 & 0.70 \\
$\begin{array}{l}\text { Customer + } \\
\text { transaction }\end{array}$ & 0.54 & 0.55 & 0.57 & 0.60 & 0.46 & 0.49 & 0.66 & 0.69 \\
$\begin{array}{l}\text { Customer + } \\
\text { transaction + novel } \\
\text { features }\end{array}$ & 0.51 & 0.59 & 0.61 & 0.69 & 0.51 & 0.61 & 0.63 & 0.55 \\
\hline
\end{tabular}

KNN=K-nearest neighbours; XGBoost=Extreme Gradient Boosted Models.

Table A5. Accuracy, F1 score, sensitivity and specificity for k-nearest neighbours and extreme gradient boosted models with a multinomial outcome.

\begin{tabular}{|c|c|c|c|c|c|c|c|c|}
\hline \multirow{2}{*}{ Dataset } & \multicolumn{2}{|c|}{ Accuracy } & \multicolumn{2}{|c|}{ F1 } & \multicolumn{2}{|c|}{ Sensitivity } & \multicolumn{2}{|c|}{ Specificity } \\
\hline & KNN & XGBoost & KNN & XGBoost & KNN & XGBoost & KNN & XGBoost \\
\hline Customer & 0.44 & 0.48 & 0.61 & 0.64 & $\begin{array}{l}\text { L: } 0.28 \\
\text { M: } 0.55 \\
H: 0.45\end{array}$ & $\begin{array}{l}\text { L: } 0.32 \\
\text { M: } 0.55 \\
H: 0.53\end{array}$ & $\begin{array}{l}\text { L: } 0.76 \\
\text { M: } 0.67 \\
\text { H: } 0.72\end{array}$ & $\begin{array}{l}\text { L: } 0.78 \\
\text { M: } 0.73 \\
\text { H: } 0.70\end{array}$ \\
\hline Customer + novel & 0.44 & 0.47 & 0.61 & 0.64 & $\begin{array}{c}\text { L: } 0.27 \\
\text { M: } 0.58 \\
\text { H: } 0.43\end{array}$ & $\begin{array}{l}\text { L: } 0.34 \\
\text { M: } 0.53 \\
\text { H: } 0.52\end{array}$ & $\begin{array}{l}\text { L: } 0.78 \\
\text { M: } 0.62 \\
\text { H: } 0.74\end{array}$ & $\begin{array}{l}\text { L: } 0.77 \\
\text { M: } 0.75 \\
\text { H: } 0.69\end{array}$ \\
\hline Customer + transaction & 0.43 & 0.50 & 0.60 & 0.67 & $\begin{array}{l}\text { L: } 0.40 \\
\text { M: } 0.47 \\
\text { H: } 0.40\end{array}$ & $\begin{array}{l}\text { L: } 0.30 \\
\text { M: } 0.68 \\
H: 0.46\end{array}$ & $\begin{array}{l}\text { L: } 0.75 \\
\text { M: } 0.71 \\
H: 0.68\end{array}$ & $\begin{array}{l}\text { L: } 0.83 \\
\text { M: } 0.66 \\
\text { H: } 0.75\end{array}$ \\
\hline $\begin{array}{l}\text { Customer + transaction } \\
+ \text { novel columns }\end{array}$ & 0.44 & 0.47 & 0.61 & 0.64 & $\begin{array}{c}\text { L: } 0.34 \\
\text { M: } 0.55 \\
\text { H: } 0.38\end{array}$ & $\begin{array}{l}\text { L: } 0.29 \\
\text { M: } 0.61 \\
\text { H: } 0.45\end{array}$ & $\begin{array}{l}\text { L: } 0.75 \\
\text { M: } 0.64 \\
\text { L: } 0.75\end{array}$ & $\begin{array}{l}\text { L: } 0.82 \\
\text { M: } 0.64 \\
\text { H: } 0.73\end{array}$ \\
\hline
\end{tabular}

KNN=K-nearest neighbours, XGBoost=Extreme Gradient Boosted Models. 